\title{
The role of temporary biventricular pacing in the cardiac surgical patient with severely reduced left ventricular systolic function
}

Rudolph F. Evonich, MD, John C. Stephens, MD, William Merhi, DO, Srinivas Dukkipati, MD, Nicholas Tepe, MD, Francis Shannon, MD, Jeffrey Altshuler, MD, Marc Sakwa, MD, Joseph Bassett, MD, Eric Hanson, MD, Judy Boura, MS, William W. O'Neill, MD, and David E. Haines, MD

From the William Beaumont Hospital, Royal Oak, Michigan.

The authors have no conflicts of interest to disclose. There was no industry funding or support for this study. The study was funded by the generous donation of Frances and Dominic Moceri.

Received for publication May 16, 2007; revisions received Nov 16, 2007; accepted for publication Nov 26, 2007.

Address for reprints: David E. Haines, MD, William Beaumont Hospital, 3601 West 13 Mile Rd, Royal Oak, MI 48073 (E-mail: DHaines@beaumont.edu).

J Thorac Cardiovasc Surg 2008;136:915-21 $0022-5223 / \$ 34.00$

Copyright $\odot 2008$ by The American Association for Thoracic Surgery

doi:10.1016/j.jtcvs.2007.11.048
Objective: The objective was to evaluate the effects of atrial synchronous biventricular pacing in postoperative patients with severe cardiomyopathy.

Methods: Atrial synchronous biventricular pacing epicardial leads were placed during cardiac surgery in patients with an ejection fraction of $30 \%$ or less. Patients were randomized to usual care pacing, the mode determined by the surgeon (excluding atrial synchronous biventricular pacing) with a preference for no pacing or atrial pacing (atrial inhibited pacing); atrial synchronous right ventricular pacing; or atrial synchronous biventricular pacing. Pacing was continued until cessation of hemodynamic support. At 12 hours postoperatively, patients were randomly tested in each mode (atrial inhibited, atrial synchronous right ventricular, and atrial synchronous biventricular pacing), and thermodilution outputs were measured.

Results: Forty subjects were randomized. Groups were similar in age (66 \pm 11 years), gender ( $85 \%$ were male), ejection fraction $(23 \% \pm 6 \%)$, QRS duration $(111 \pm 30 \mathrm{~ms})$, and surgical indication. There was no difference in stroke index or cardiac index at 12 hours, duration of inotropic or intra-aortic balloon pump support, intensive care unit, or hospital length of stay. On comparative crossover testing, stroke volume was similar with atrial inhibited pacing and atrial synchronous biventricular pacing $(59.3 \pm$ 13.4 vs $57 \pm 12.1$, respectively, $P=$ not significant); however, atrial synchronous right ventricular pacing was inferior $(56 \pm 12.9, P<.05$ for comparison with atrial inhibited pacing). When compared with atrial inhibited pacing, atrial synchronous biventricular pacing showed a positive response in $17 \%$ of subjects (increase in stroke volume $\geq 5 \%$ ), whereas $41 \%$ had a $5 \%$ or greater decrease in stroke volume.

Conclusion: Pacing mode affects stroke volume in patients with severe cardiomyopathy. Atrial synchronous biventricular pacing was helpful in a minority, but in $41 \%$ it compromised stroke volume.

$\mathrm{L}$ eft ventricular dysfunction is an independent risk factor for increased mortality in patients undergoing coronary artery bypass grafting (CABG). ${ }^{1}$ When compared with patients with normal ejection fractions, these patients more frequently require inotropic or intra-aortic balloon pump (IABP) support and have longer lengths of hospital stay. ${ }^{2,3}$ Given the widespread use and effectiveness of percutaneous coronary revascularization, the contemporary patient who undergoes cardiac surgery is more likely to present later in the course of disease and have severe cardiomyopathy with associated comorbidities. Conduction system abnormalities after cardiac surgery are common and may portend a negative prognosis. ${ }^{4,5}$ Accordingly, most patients who undergo $\mathrm{CABG}$ receive temporary atrial and right ventricular (RV) epicardial pacing leads at the time of surgery. Studies in chronic pacing have demonstrated worse outcomes with RV pacing compared with native conduction in patients receiving implantable defibrillators for primary prevention of sudden cardiac 


\section{Abbreviations and Acronyms}

AAI = atrial inhibited

$\mathrm{ABiV}=$ atrial synchronous biventricular

$\mathrm{ARV}=$ atrial synchronous right ventricular

$\mathrm{AV}=$ atrioventricular

$\mathrm{CABG}=$ coronary artery bypass grafting

IABP $=$ intra-aortic balloon pump

$\mathrm{RV}=$ right ventricular death ${ }^{6}$ presumably because RV pacing induces ventricular dyssynchrony, resulting in reduced cardiac output. In contrast, acute and chronic hemodynamic benefits have been demonstrated with atrial synchronous biventricular (ABiV) pacing in patients with severely impaired left ventricular systolic function and a prolonged QRS duration. ${ }^{7-12}$ Permanent $\mathrm{ABiV}$ pacing confers additional clinical benefits, including increased exercise tolerance, quality of life, and left ventricular ejection fraction, and reduced heart failure hospitalization. ${ }^{9,12}$

On the basis of these observations, it has been suggested that temporary $\mathrm{ABiV}$ pacing may further improve the postoperative management of the patient with severe cardiomyopathy after cardiovascular surgery, ${ }^{13-17}$ and some authors recommend routine $\mathrm{ABiV}$ pacing for all patients with severe cardiomyopathy undergoing cardiac surgery. ${ }^{18}$ However, the role of $\mathrm{ABiV}$ pacing after cardiac surgery has been evaluated in only small numbers of patients post-CABG, and there are scant data pertaining to those with left ventricular ejection fractions less than $30 \%$. The purpose of this study was to determine whether $\mathrm{ABiV}$ pacing would improve postoperative hemodynamics, lessen the requirement for IABP or inotropic support, decrease length of hospital stay, or decrease mortality in this specific patient population.

\section{Materials and Methods Patient Selection}

All non-urgent coronary bypass and valvular heart surgeries performed at William Beaumont Hospital, Royal Oak, Michigan, between October 20, 2004, and January 23, 2006, were screened for eligibility. A total of 1204 cardiac surgeries were performed at William Beaumont Hospital and screened for eligibility during the enrollment period. Eighty-one surgeries (7\%) were performed in patients with a preoperative ejection fraction of $30 \%$ or less. Twenty-eight patients were excluded from enrollment because of the presence of one of the following: urgent surgery (18 patients); enrollment in competing clinical trial (5 patients); presence of permanent biventricular pacemaker ( 2 patients); or inability to provide informed consent ( 3 patients). Thirteen patients refused consent. The remaining 40 subjects comprise the study population.

\section{Study Design}

The study protocol was approved and monitored by the William Beaumont Hospital Human Investigations Committee. Patients were screened 1 to 7 days before cardiac surgery and underwent informed consent at that time. Cardiac surgeries were performed in accordance with accepted surgical practice. Before weaning from cardiopulmonary bypass, temporary epicardial pacing wires were sutured into the right atrium, right ventricle, and left ventricle. Subjects were then randomized in the operating room to 1 of 3 treatment arms: usual care pacing, atrial synchronous right ventricular (ARV) pacing, or ABiV. Postoperative hemodynamic and clinical data were recorded for each study subject as detailed below. The primary study end point was intensive care unit length of stay. Secondary end points were hospital length of stay, mortality, and hemodynamic response to each pacing modality. Because of dropout in the 2 active pacing arms, on-treatment analysis was performed in addition to intention-to-treat analysis.

\section{Pacing Protocol}

A DDD temporary external pulse generator (Model 5346, Medtronic, Inc, Minneapolis, Minn) was used with bipolar epicardial pacing wires (Model 6495, Medtronic, Inc) positioned on the right atrium, mid-right ventricle, and mid-left ventricle (left circumflex distribution). An adaptor was plugged into the ventricular output of the temporary pacemaker generator enabling simultaneous pacing of the right and left ventricles in those patients randomized to the ABiV pacing modality. Pacing thresholds were tested to confirm lead function and capture. Depending on the personal preference of the operating surgeon, usual care pacing consisted of no pacing or atrial pacing (AAI) if atrioventricular (AV) conduction was intact. For ARV, the mode was DDD, heart rate was programmed to 85 beats/min or more, and AV delay was 150 ms. ABiV was performed in the DDD mode with a heart rate of 85 beats/min or more and an AV delay programmed to $150 \mathrm{~ms}$ or less to ensure ventricular pacing. Pacing with the assigned modality was continued until the cessation of vasopressors, inotropes, or IABP support.

\section{Hemodynamic Data Collection and Analysis}

Hemodynamic testing with AAI, ARV, and ABiV pacing was performed in each subject at 12 hours postoperatively (or when clinically feasible) at a time when the doses of vasopressors, inotropes, or degree of IABP support could be safely maintained at a constant level for the 40-minute duration of testing. The sequence of pacing modes during hemodynamic testing was random. The use of new medications, transfusion of blood products, or change in fluid administration or ventilator setting during the testing sequence was avoided if at all possible.

The standard minimum paced heart rate was 85 beats $/ \mathrm{min}$. If the subject's sinus rate was greater than this rate, the lower rate limit of the pacer was increased to 10 beats/min above the sinus rate. The AV interval was adjusted to ensure active ventricular pacing and kept constant during the ARV and ABiV pacing tests. After 5 minutes of pacing in each modality, thermodilution cardiac outputs were measured by injecting $10 \mathrm{~mL}$ of normal saline through a 7F Swan-Ganz catheter (Edwards Life Sciences LLC, Irvine, Calif) positioned in the pulmonary artery. Five outputs were measured for each pacing modality. The high and low values were discarded, with the remaining 3 averaged for reporting. Five-minute delays were instituted after each change in pacing modality before repeating thermodilution cardiac output measurements. 


\section{Clinical Data Collection and Analysis}

Clinical, operative, and in-hospital data were recorded in a computer database. Clinical variables recorded were gender, age, height, weight, race, medical history, medications, ejection fraction, pulse repetition interval, QRS duration, and conduction disturbance. Operative data recorded included surgical date, indication, procedure(s) performed, and duration of cardiopulmonary bypass. Inhospital variables were intensive care unit length of stay, inpatient length of stay, mortality, postoperative complications, and duration of pharmacologic or mechanical hemodynamic support and mechanical ventilation. Left ventricular ejection fractions were determined by 2 independent readers using one of the following imaging modalities: left-sided heart catheterization with quantitative left ventriculography (QuantCor LVA, Siemens, Forchheim, Germany); transthoracic or transesophageal echocardiography (General Electric Vivid 7, Milwaukee, Wis; Siemens Sequoia; or Hewlett Packard IE33, Wilmington, Del); multiple gated acquisition scan; quantitative cardiac cine magnetic resonance imaging (Argus, Siemens). Patients with ejection fractions of $30 \%$ or less by the most recent determination were included in the study.

\section{Statistics}

The calculated sample size was 400 patients. A pilot phase of 40 subjects is reported in the present study, which was powered to detect a $15 \%$ acute hemodynamic treatment effect with a power of 0.95 at a significance of .05. Statistical analysis was performed using SAS 9.1 (Cary, NC). Randomization was accomplished by prospective generation of a 3-number random number table and applying the group assignment in a blinded fashion sequentially to each patient randomized. Data were analyzed on an intention-to-treat basis. Normal distributed repeated measures were analyzed with analysis of variance. Non-normal continuous variables were analyzed with the Kruskal-Wallis nonparametric test. Data are reported as mean \pm 1 standard deviation followed by the median. Categoric variables were compared with a chi-square test when appropriate; otherwise, a Fisher exact test was used.

\section{Results}

Patient Demographics

The clinical characteristics of the patient population are summarized in Table 1. The majority of patients enrolled were male with a mean age $66 \pm 11$ years. The mean left ventricular ejection fraction was $23 \% \pm 6 \%$, and $53 \%$ had a history of congestive heart failure. The 3 treatment groups were similar in age, gender, preoperative ejection fraction, New York Heart Association class, QRS duration, preoperative medications, surgical indication, cardiopulmonary bypass duration, and surgical procedure(s) performed. The mean QRS duration was $111 \pm 30 \mathrm{~ms}$. Eleven patients had a significant intraventricular conduction delay on the preoperative electrocardiogram: left bundle branch block in 4 patients, right bundle branch block in 5 patients, and nonspecific intraventricular conduction defect in 2 patients. Of the surgical procedures performed, 23 were $\mathrm{CABG}, 9$ were combined $\mathrm{CABG}$ and valve repair or replacement, 7 were isolated valve repairs or replacements, and 1 was left ventricular

TABLE 1. Clinical characteristics of study population

\begin{tabular}{|c|c|c|c|c|c|}
\hline & Entire cohort & Usual care & ARV pacing & ABiV pacing & $P$ value \\
\hline No. & 40 & 13 & 14 & 13 & \\
\hline Age (y) & $66 \pm 11(66.5)$ & $68 \pm 8(68)$ & $63 \pm 13(64)$ & $66 \pm 13(66)$ & .69 \\
\hline Sex (male) & $34(85 \%)$ & $10(77 \%)$ & $14(100 \%)$ & $10(77 \%)$ & .16 \\
\hline $\mathrm{EF}$ & $23 \pm 6(25)$ & $24 \pm 7(25)$ & $23 \pm 6(23)$ & $23 \pm 5(23)$ & .89 \\
\hline QRS (msec) & $111 \pm 30$ (99) & $116 \pm 37(100)$ & $99 \pm 13(99)$ & $119 \pm 33(103)$ & .50 \\
\hline LBBB & $4(10 \%)$ & $2(15 \%)$ & 0 & $2(15 \%)$ & .37 \\
\hline RBBB & $5(12.5 \%)$ & $2(15 \%)$ & 0 & $3(23 \%)$ & .17 \\
\hline IVCD & $2(5 \%)$ & 0 & $2(14 \%)$ & 0 & .32 \\
\hline Hypertension & $28(70 \%)$ & $11(85 \%)$ & $9(64 \%)$ & $8(62 \%)$ & .47 \\
\hline Diabetes mellitus & $16(40 \%)$ & $5(38 \%)$ & $6(43 \%)$ & $5(38 \%)$ & .96 \\
\hline Hyperlipidemia & $26(65 \%)$ & $6(46 \%)$ & $11(79 \%)$ & $9(69 \%)$ & .25 \\
\hline PVD & $6(15 \%)$ & $2(15 \%)$ & $2(14 \%)$ & $2(15 \%)$ & 1.00 \\
\hline $\mathrm{CHF}$ & $21(53 \%)$ & $8(62 \%)$ & $5(36 \%)$ & $8(62 \%)$ & .30 \\
\hline Previous MI & $24(60 \%)$ & $9(69 \%)$ & $7(50 \%)$ & $8(62 \%)$ & .59 \\
\hline COPD & $7(18 \%)$ & $2(15 \%)$ & $2(14 \%)$ & $3(23 \%)$ & .88 \\
\hline CABG & $32(80 \%)$ & $8(62 \%)$ & $14(100 \%)$ & $10(77 \%)$ & .03 \\
\hline Mitral regurgitation & $16(40 \%)$ & $3(23 \%)$ & $8(57 \%)$ & $5(38 \%)$ & .19 \\
\hline Aortic insufficiency & $9(23 \%)$ & $2(15 \%)$ & $2(14 \%)$ & $3(23 \%)$ & .88 \\
\hline Aortic stenosis & $10(25 \%)$ & $4(31 \%)$ & $3(21 \%)$ & $3(23 \%)$ & .90 \\
\hline Valve surgery & $16(40 \%)$ & $5(38 \%)$ & $7(50 \%)$ & $4(31 \%)$ & 0.59 \\
\hline
\end{tabular}

$A R V$, Atrial synchronous right ventricular; $A B i V$, atrial synchronous biventricular; $C A B G$, coronary artery bypass graft surgery; $C H F$, congestive heart failure; $C O P D$, chronic obstructive coronary disease; $E F$, ejection fraction; $I V C D$, intraventricular conduction defect; $L B B B$, left bundle branch block; $M I$, myocardial infarction; $P V D$, peripheral vascular disease; $R B B B$, right bundle branch block. Data are expressed as means \pm standard deviation (median value or percentage). 
aneurysmectomy. All patients underwent surgery on cardiopulmonary bypass.

\section{Hemodynamic Testing}

Twenty-nine of the 40 subjects $(73 \%)$ completed hemodynamic testing in all 3 pacing modalities approximately 12 hours after surgery. Testing was not completed because of complete heart block in 3 subjects, lead capture failures in 3 subjects, atrial fibrillation in 2 subjects, painful pacing stimuli in 2 subjects, and inadvertent removal of the pulmonary artery catheter before testing in 1 subject. The results of this testing are shown in Table 2. For this study population, $\mathrm{ABiV}$ pacing mode yielded hemodynamic results similar to the AAI pacing mode, including stroke volume $(57.0$ vs $59.3 \mathrm{~mL}, P=$ not significant), cardiac output (5.4 vs $5.5 \mathrm{~L} /$ min, $P=$ not significant), and left ventricular stroke work in$\operatorname{dex}(23.8$ vs $24.5 \mathrm{gm}-\mathrm{m} /$ beat, $P=$ not significant). Five of 29 patients $(17 \%)$ had more than $5 \%$ improvement in stroke volume, cardiac output, or left ventricular stroke work index with ABiV compared with AAI pacing; however, 12 of 29 patients (41\%) experienced a greater than 5\% decrease in hemodynamics (Figure 1). An association between hemodynamic benefit from the biventricular pacing mode and baseline QRS duration, New York Heart Association class, severity of mitral regurgitation, or surgical indication was not observed. A trend toward lower baseline left ventricular ejection fraction was seen in subjects with a positive response to the $\mathrm{ABiV}$ pacing mode $(20 \% \pm 8 \%)$ versus those with a negative response $(23 \% \pm 6 \%)$, but this did not achieve statistical significance.

When ARV pacing mode was compared with AAI pacing mode, a decrease in hemodynamic function was observed with single-site RV pacing. Stroke volume (56.0 vs 59.3 $\mathrm{mL}, P<.05$ ), cardiac output (5.3 vs $5.5 \mathrm{~L} / \mathrm{min}, P<.05$ ), and left ventricular stroke work index (22.9 vs $24.5 \mathrm{gm}-\mathrm{m} /$ beat, $P<.05)$ were all reduced. Furthermore, $48 \%$ of sub- jects demonstrated an adverse hemodynamic response to ARV pacing compared with AAI pacing, compared with $17 \%$ of patients who improved in the ARV mode (Figure 1). There was no association between response to ARV pacing and baseline left ventricular ejection fraction, QRS duration, New York Heart Association class, severity of mitral regurgitation, or surgical indication. No significant differences in acute hemodynamics could be discerned between ARV and ABiV pacing modes.

\section{Outcomes of Pacing}

Thirty of 40 patients maintained the assigned pacing modality until completion of the pacing period (cessation of vasopressors, inotropes, or IABP support). Ten subjects had early crossover or discontinuation of pacing because of failure of capture in the atrial or ventricular lead $(n=5)$, onset of atrial fibrillation with rapid ventricular response $(\mathrm{n}=2)$, hypotension with ventricular pacing $(\mathrm{n}=2)$, and painful muscle stimulation $(\mathrm{n}=1)$. One patient assigned to the ARV pacing group died of cardiogenic shock complicated by ventilator-associated pneumonia and sepsis on hospital day 25. Of the 13 subjects randomized to the usual care pacing arm, 6 received no pacing, 4 received atrial pacing, and 3 received AV sequential pacing because of postoperative heart block. Outcomes by group assignment are listed in Table 3. Because of the high crossover rate, both intention-to-treat and on-treatment analyses were performed. Among the 3 pacing modes, there were no statistically significant differences in the predetermined primary or secondary end points of intensive care unit length of stay, hospital length of stay, mortality, or hemodynamic parameters measured 12 hours postoperatively for either analysis.

\section{Discussion}

Anecdotal observations have led some to consider routine biventricular pacing for patients with severe cardiomyopathy

TABLE 2. Acute hemodynamic outcomes during crossover testing

\begin{tabular}{|c|c|c|c|c|}
\hline & Usual care & ARV pacing & ABiV pacing & $P$ value \\
\hline Heart rate $(\mathrm{bpm})$ & $94 \pm 10(91)$ & $94 \pm 8(91)$ & $95 \pm 8(94)$ & .90 \\
\hline Mean blood pressure $(\mathrm{mm} \mathrm{Hg})$ & $75 \pm 11(74)$ & $78 \pm 15(75)$ & $78 \pm 12(78)$ & .52 \\
\hline Mean PAP $(\mathrm{mm} \mathrm{Hg})$ & $25 \pm 5(25)$ & $25 \pm 5(25)$ & $25 \pm 5(24)$ & .99 \\
\hline PCWP (mm Hg) & $18 \pm 5(18)$ & $18 \pm 5(17)$ & $18 \pm 5(18) .3(22.6)$ & .87 \\
\hline Stroke volume $(\mathrm{mL})$ & $56.0 \pm 13.7(54.9)^{*}$ & $57.0 /-12.4(55.4)$ & $59.3 \pm 11.9(58.1)$ & .92 \\
\hline Cardiac output (L/min) & $5.3 \pm 1.1(5.3)^{*}$ & $5.4 \pm 0.9(5.2)$ & $5.5 \pm 1.0(5.4)$ & .97 \\
\hline Cardiac index $\left(\mathrm{L} / \mathrm{min} / \mathrm{m}^{2}\right)$ & $2.7 \pm 0.6(2.6)$ & $2.7 \pm 0.4(2.6)$ & $2.8 \pm 0.4(2.7)$ & .83 \\
\hline SVRI & $2176 \pm 1350$ & $2277 \pm 1432$ & $1971 \pm 391$ & .56 \\
\hline Stroke index & $29.6 \pm 6.0(28.2)$ & $29.0 \pm 5.5(27.9)$ & $29.2 \pm 5.5(27.9)$ & .97 \\
\hline LVSWI (gm-m/beat) & $22.9 \pm 7.2(21.2)^{*}$ & $23.8 \pm 7.7(22.8)$ & $24.5 \pm 7.3$ & .57 \\
\hline
\end{tabular}

$A R V$, Atrial synchronous right ventricular; $A B i V$, atrial synchronous biventricular; $L V S W I$, left ventricular stroke work index; $P A P$, pulmonary artery pressure; $P C W P$, pulmonary capillary wedge pressure; $S V R I$, systemic vascular resistance index. ${ }^{*} P<.05$ ARV versus AAl pacing mode. Data are expressed as means \pm standard deviation (median value). 


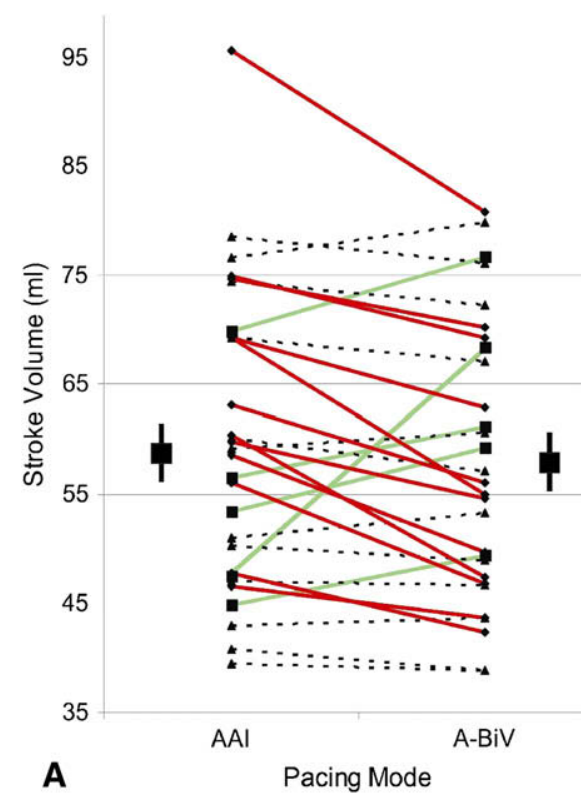

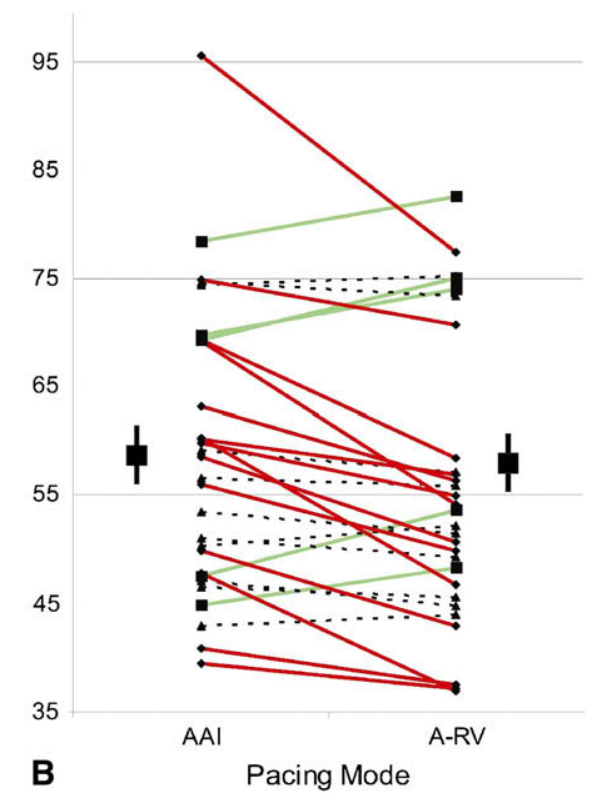

Figure 1. Stroke volume versus pacing mode during crossover hemodynamic testing. A, AAl pacing compared with ABiV pacing. B, AAl pacing compared with ARV pacing. Each symbol represents an individual study subject's stroke volume in each pacing mode. Subjects who had no significant change $(<5 \%)$ (triangles with dotted lines), subjects who had a decrease of $\mathbf{5 \%}$ or more (diamonds with solid black lines), and subjects who had a $5 \%$ or greater improvement in hemodynamic parameters with crossover to the alternate pacing mode (squares with grey lines) are shown. Mean \pm standard error of the mean (large squares). undergoing cardiac surgery. Gaudiani and colleagues ${ }^{18}$ report using $\mathrm{ABiV}$ pacing "without difficulty in all high risk cases for the past two years" and recommends widespread use in "all high-risk cardiac surgery patients." To test this hypothesis, the present pilot study sought to compare 3 temporary pacing modes in a cohort of patients with significant left ventricular dysfunction who underwent cardiac surgery. The results failed to demonstrate consistent superiority of ABiV when broadly applied to the postoperative management of these patients. In fact, $\mathrm{ABiV}$ resulted in diverse hemodynamic responses among individuals in this study population, with $17 \%$ of patients demonstrating improved hemodynamics and $41 \%$ showing worsened measurements. With hemodynamic testing performed in each pacing modality in the early postoperative period, we were unable to demonstrate statistically significant differences in any hemodynamic parameters among the 3 groups. However, ARV resulted in worse hemodynamics overall, with a decline in function in more than one half of the population studied in this mode. The overall outcomes were similar for all 3 groups in pacing mode assignment and pacing mode received. No conclusions could be drawn about the clinical outcomes of this study because it was not adequately powered to do so.

\section{Previous Studies}

The observations from the present pilot trial are not consistent with those reported in several small nonrandomized surgical studies that suggested biventricular pacing favorably affects postoperative hemodynamics in patient undergoing cardiac surgery. ${ }^{13-15}$ The disparate findings among studies

TABLE 3. Clinical outcome based on intention-to-treat analysis of study group assignment

\begin{tabular}{|c|c|c|c|c|c|}
\hline & Entire cohort & Usual care & ARV pacing & ABiV pacing & $P$ value \\
\hline No. & 40 & 13 & 14 & 13 & \\
\hline ICU LOS (h) & $87 \pm 92(63)$ & $71 \pm 47(70)$ & $101 \pm 141(45)$ & $89 \pm 58(66)$ & .62 \\
\hline Heart rate & $94 \pm 9(94)$ & $93 \pm 7(96)$ & $95 \pm 13(92)$ & $95 \pm 7(94)$ & .46 \\
\hline Stroke index & $29 \pm 5(28)$ & $29.3 \pm 4.8(29.4)$ & $28.7 \pm 5.8(28)$ & $29 \pm 5.6(27.7)$ & .84 \\
\hline Cardiac index & $2.72 \pm 0.5(2.67)$ & $2.70 \pm 0.3(2.7)$ & $2.73 \pm 0.7(2.55)$ & $2.74 \pm 0.5(2.75)$ & .77 \\
\hline Duration of inotropic support (h) & $39 \pm 61(16)$ & $43 \pm 84(9)$ & $48 \pm 64(17)$ & $25 \pm 21(17)$ & .35 \\
\hline IABP & $11(28 \%)$ & $3(23 \%)$ & $3(21 \%)$ & $5(38 \%)$ & .67 \\
\hline Duration IABP $(\mathrm{h})$ & $46 \pm 50(27)$ & $39 \pm 18(46)$ & $88 \pm 92(49)$ & $25 \pm 6(23)$ & .30 \\
\hline Duration ventilation $(\mathrm{h})$ & $37 \pm 88(15)$ & $21 \pm 22(12)$ & $60 \pm 147(15)$ & $27 \pm 23(24)$ & .38 \\
\hline Hospital LOS (d) & $14.4 \pm 7.5(13)$ & $16 \pm 9(17)$ & $15 \pm 7(16)$ & $12 \pm 7(11)$ & .22 \\
\hline Postoperative AF & $15(38 \%)$ & $7(54 \%)$ & $6(43 \%)$ & $4(31 \%)$ & .49 \\
\hline Mortality & $1(2.5 \%)$ & 0 & $1(7 \%)$ & 0 & \\
\hline
\end{tabular}

$A B i V$, Atrial synchronous biventricular; $A R V$, atrial synchronous right ventricular; $A F$, atrial fibrillation; $I A P B$, intra-aortic balloon pump; $I C U$, intensive care unit; $L O S$, length of stay. Data are expressed as means \pm standard deviation (median value or percentage). 
are likely due to dissimilar study populations, pacing protocols, and methodologies for hemodynamic and clinical data acquisition. Foster and colleagues ${ }^{13}$ demonstrated a $7.6 \%$ increase in cardiac index with biventricular pacing when compared with AAI pacing. There were only 18 subjects in the study, and most had preserved left ventricular systolic function. Subjects were excluded if the cardiac index was less than $2.0 \mathrm{~L} / \mathrm{min} / \mathrm{m}^{2}$ or if IABP support was required. Weisse and colleagues ${ }^{14}$ compared pacing modalities in 22 patients with a left ventricular ejection fraction less than $40 \%$ and new bundle branch block after cardiac surgery. Among the 12 patients with left bundle branch block, biventricular pacing improved the cardiac index by $14.5 \%$ when compared with AAI pacing. In the 10 patients with right bundle branch block, $\mathrm{ABiV}$ pacing improved cardiac index by $14.1 \%$ versus AAI pacing. The present cohort represented a more ill population of patients than previously tested. The mean left ventricular ejection fraction was $23 \%, 53 \%$ had a history of heart failure, and $28 \%$ required IABP support.

Successful resynchronization is dependent on optimal placement of the pacing electrodes. ${ }^{19}$ If the site selected for left ventricular pacing is diseased, particularly with transmural infarct scar, the electrical conduction emanating from that site may be slow, and the left ventricular activation, in turn, may be delayed. ${ }^{20,21}$ The present pacing protocol required epicardial leads to be positioned in the mid-right ventricle and mid-left ventricle along the distribution of the left circumflex coronary artery. This widely spaced electrode configuration has been demonstrated in previous work to result in a greater likelihood of synchronous ventricular contraction. ${ }^{22}$ In contrast, the previous studies used paraseptal left ventricular and RV lead placement. ${ }^{14,15}$ It might be anticipated that placement of paraseptal left ventricular and $\mathrm{RV}$ leads close to one another would not result in true synchronous pacing in all individuals. The study by Saxon and colleagues ${ }^{15}$ compared asynchronous ventricular pacing modes with sinus rhythm and did so intraoperatively in an open chest. This less-physiologic methodology makes it difficult to draw strong conclusions from these observations.

\section{Limitations}

The present study was a pilot study by design and therefore did not have a large enough population to achieve adequate statistical power to detect small differences in clinical outcomes among the 3 groups. In addition, the high crossover rate during pacing makes it difficult to draw conclusions about the clinical end points based on the intention-to-treat analysis. Nonetheless, this work represents the largest randomized trial to date comparing postoperative hemodynamics with different pacing modalities in this patient population. Our patients were intentionally not selected on the basis of electrocardiographic or echocardiographic parameters of dyssynchrony. We chose to include all patients with an $\mathrm{EF}$ of $30 \%$ or less to make this study more widely applicable. As a result, only a minority of subjects in this population had preexisting bundle branch block. Our findings may have been different had the participants had evidence of dyssynchrony. The final AV interval was not recorded prospectively, and the AV and $\mathrm{VV}$ timing intervals were not optimized. Differences in AV intervals among groups may have affected acute hemodynamics and late outcomes, and AV and VV timing optimization could have improved response to BiV pacing. ${ }^{23,24}$ The hours postsurgery are a dynamic period; testing was performed by protocol 12 hours postoperatively, but biventricular pacing might have shown different acute hemodynamic results had the randomized testing been performed at a different time interval.

\section{Conclusions}

Patients with significant left ventricular dysfunction undergoing cardiac surgery did not show substantive benefit with ABiV compared with atrial pacing alone, but atrial synchronous RV pacing led to an acute decrease in hemodynamics. Individual hemodynamic responses to biventricular pacing were highly variable. Although biventricular pacing might result in hemodynamic advantage in some patients, routine biventricular pacing in all high-risk patients undergoing cardiac surgery could result in more harm than benefit. Therefore, normal sinus rhythm or atrial pacing alone is preferable to any ventricular pacing if the heart rate can be maintained adequately. If heart block results in a need for ventricular pacing, then ABiV may be preferable to RV pacing. Further larger trials will need to be performed to elucidate these questions.

\section{References}

1. Alderman EL, Fisher LD, Litwin P, Kaiser GC, Myers WO, Maynard C, et al. Results of coronary artery surgery in patients with poor left ventricular function (CASS). Circulation. 1983;68:785-95.

2. Antunes PE, Ferrao de Oliveira JM, Antunes MJ. Coronary surgery with non-cardioplegic methods in patients with advanced left ventricular dysfunction: immediate and long term results. Heart. 2003;89:427-31.

3. Trachiotis GD, Weintraub WS, Johnston TS, Jones EL, Guyton RA, Craver JM. Coronary artery bypass grafting in patients with advanced left ventricular dysfunction. Ann Thorac Surg. 1998;66:1632-9.

4. Mustonen P, Hippelainen M, Vanninen E, Rehnberg S, TenhunenEskelinen M, Hartikainen J. Significance of coronary artery bypass grafting-associated conduction defects. Am J Cardiol. 1998;81:558-63.

5. Pires LA, Wagshal AB, Lancey R, Huang SK. Arrhythmias and conduction disturbances after coronary artery bypass graft surgery: epidemiology, management, and prognosis. Am Heart J. 1995; 129:799-808.

6. Wilkoff BL, Cook JR, Epstein AE, Greene L, Hallstrom AP, Hsia H, et al, for the DAVID trial investigators. Dual-chamber pacing or ventricular backup pacing in patients with an implantable defibrillator. JAMA. 2002;288:3115-23.

7. Blanc JJ, Etienne Y, Gilard M, Mansourati J, Munier S, Boschat J, et al. Evaluation of different ventricular pacing sites in patients with severe heart failure. Results of an acute hemodynamic study. Circulation. 1997;96:3273-7.

8. Leclercq C, Cazeau S, Breton HL, Ritter P, Mabo P, Gras D, et al. Acute hemodynamic effects of biventricular DDD pacing in patients with end-stage heart failure. J Am Coll Cardiol. 1998;32:1825-31.

9. Cazeau S, Leclercq C, Lavergne T, Walker S, Varma C, Linde C, et al, on behalf of the multisite stimulation in cardiomyopathy (MUSTIC) 
study group. Effects of multisite biventricular pacing in patients with heart failure and intraventricular conduction delay. $N$ Engl $J$ Med. 2001;334:873-80.

10. Linde C, Leclercq C, Rex S, Garrigue S, Lavergne T, Cazeau S, et al, for the multisite stimulation in cardiomyopathy (MUSTIC) study investigators. Long-term benefits of biventricular pacing in congestive heart failure: results from the multisite stimulation in cardiomyopathy (MUSTIC) study. J Am Coll Cardiol. 2002;40:111-8.

11. Cleland JG, Daubert JC, Erdmann E, Freemantle N, Gras D, Kappenberger L, et al, for the Cardiac Resynchronization-Heart Failure (CARE-HF) study investigators. The effect of cardiac resynchronization on morbidity and mortality in heart failure. N Engl J Med. 2005;352: 1539-49.

12. Abraham WT, Fisher WG, Smith AL, Delurgio DB, Leon AR, Loh E, et al, for the MIRACLE study group. Cardiac resynchronization in chronic heart failure. $N$ Engl J Med. 2002;346:1845-53.

13. Foster AH, Gold MR, McLaughlin JS. Acute hemodynamic effects of atrio-biventricular pacing in humans. Ann Thorac Surg. 1995;59: 294-300.

14. Weisse U, Isgro F, Werling C, Lehmann A, Saggau W. Impact of atrio-biventricular pacing to poor left-ventricular function after CABG. Thorac Cardiovasc Surg. 2002;41:131-5.

15. Saxon LA, Kerwin WF, Cahalan MK, Kalman JM, Olgin JE, Foster E, et al. Acute effects of intraoperative multisite ventricular pacing on left ventricular function and activation/contraction sequence in patients with depressed ventricular function. J Cardiovasc Electrophysiol. 1998;9: 13-21.

16. Tanaka H, Okishige K, Mizuno T, Kuriu K, Itoh F, Shimizu M, et al. Temporary and permanent biventricular pacing via left ventricular epicardial leads implanted during primary cardiac surgery. Jpn J Thorac Cardiovasc Surg. 2002;50:284-9.

17. Mizuno T, Tanaka H, Makita S, Tabuchi N, Arai H, Sunamori M. Biventricular pacing with coronary bypass and Dor's ventriculoplasty. Ann Thorac Surg. 2003;75:998-9.

18. Gaudiani VA, Castro LJ, Fisher AL. Biventricular pacing during cardiac operations. Heart Surg Forum. 2003;6:E126-8.

19. Rossillo A, Verma A, Saad EB, Corrado A, Gasparini G, Marrouche NF, et al. Impact of coronary sinus lead position on biventricular pacing: mortality and echocardiographic evaluation during long-term followup. J Cardiovasc Electrophysiol. 2004;15:1120-5.

20. Lambiase PD, Rinaldi A, Hauck J, Mobb M, Elliott D, Mohammad S, et al. Non-contact left ventricular endocardial mapping in cardiac resynchronisation therapy. Heart. 2004;90:44-5.

21. Bleeker GB, Kaandorp TA, Lamb HJ, Boersma E, Steendijk P, de Roos A, et al. Effect of posterolateral scar tissue on clinical and echocardiographic improvement after cardiac resynchronization therapy. Circulation. 2006;113:969-76.

22. Butter C, Auricchio A, Stellbrink C, Fleck E, Ding J, Yu Y, et al, on behalf of the PATH-CHF II Study Group. Effect of Resynchronization therapy stimulation site on the systolic function of heart failure patients. Circulation. 2001;104:3026-9.

23. Kerlan JE, Sawhney NS, Waggoner AD, Chawla MK, Garhwal S, Osborn JL, et al. Prospective comparison of echocardiographic atrioventricular delay optimization methods for cardiac resynchronization therapy. Heart Rhythm. 2006;3:148-54.

24. Vanderheyden M, De Backer T, Rivero-Ayerza M, Geelen P, Bartunek J, Verstreken S, et al. Tailored echocardiographic interventricular delay programming further optimizes left ventricular performance after cardiac resynchronization therapy. Heart Rhythm. 2005;2:1066-72. 